\title{
Liquid-based gating mechanism with tunable multiphase selectivity and antifouling behaviour
}

\section{Citation}

Hou, Xu, Yuhang Hu, Alison Grinthal, Mughees Khan, and Joanna Aizenberg. 2015. Liquid-Based Gating Mechanism with Tunable Multiphase Selectivity and Antifouling Behaviour. Nature 519, no. 7541: 70-73. doi:10.1038/nature14253. .

\section{Published Version}

doi:10.1038/nature14253

\section{Permanent link}

http://nrs.harvard.edu/urn-3:HUL.InstRepos:27657493

\section{Terms of Use}

This article was downloaded from Harvard University's DASH repository, and is made available under the terms and conditions applicable to Other Posted Material, as set forth at http:// nrs.harvard.edu/urn-3:HUL.InstRepos:dash.current.terms-of-use\#LAA

\section{Share Your Story}

The Harvard community has made this article openly available.

Please share how this access benefits you. Submit a story.

\section{Accessibility}




\title{
Fluid-Based Gating Mechanism with Tunable Multiphase Selectivity and Antifouling Behavior
}

\author{
Xu Hou, ${ }^{1,2}$ Yuhang Hu, ${ }^{1}$ Alison Grinthal,,${ }^{1}$ Mughees Khan, ${ }^{2}$ Joanna Aizenberg ${ }^{1,2} *$ \\ ${ }^{1}$ School of Engineering and Applied Sciences, Harvard University, Cambridge, MA 02138, USA \\ ${ }^{2}$ Wyss Institute for Biologically Inspired Engineering, Harvard University, Cambridge, MA 02138, USA
}

*To whom correspondence should be addressed. Email: jaiz@seas.harvard.edu

Living organisms make extensive use of micro- and nanometer-sized pores as gatekeepers for controlling the movement of fluids, vapors and solids between complex environments. The ability of such pores to coordinate multiphase transport, in a highly selective and subtly triggered fashion and without clogging, has inspired interest in synthetic gated pores for applications ranging from fluid processing to $3 \mathrm{D}$ printing and labon-chip systems $1,2,3,4,5,6,7,8,9,10$. But although specific gating and transport behaviors have been realized by precisely tailoring pore surface chemistries and pore geometries ${ }^{6,11-17}$, a single system capable of selectively handling and controlling complex multiphase transport has remained a distant prospect, and fouling is nearly inevitable ${ }^{11,12}$. Here, we introduce a gating mechanism that uses a capillary-stabilized fluid to seal pores in the closed state, and reversibly and rapidly reconfigures it under pressure to create a non-fouling, fluid-lined pore in the open state. Theoretical modeling and experiments demonstrate that for each transport substance, the gating threshold - the pressure needed to open pores - can be rationally tuned over a wide pressure range. This allows us to realize in one system differential response profiles for a variety of liquids and gases, even letting liquids flow through the pore while preventing gas escape. These capabilities allow us to dynamically modulate gas/liquid sorting in a microfluidic flow and to separate a three-phase air/water/oil mixture, with the fluid lining ensuring sustained antifouling behavior. Because the liquid gating strategy enables efficient long-term operation and can be applied to a variety of pore structures, membrane materials and micro- as well as macro-scale fluid systems, we expect it to prove useful in a wide range of applications.

Our hypothesis that a liquid-filled pore could provide a unified gating strategy derives from the idea that a liquid stabilized inside a micropore offers a unique combination of dynamic and interfacial behaviors, and is inspired by nature's use of fluids as reconfigurable gates. Microscale stomata and xylem control air, water, and microbe exchange in plants by using fluid to mechanically reconfigure the pore ${ }^{18}$. The nuclear pore is directly lined with disordered fluidlike proteins that have been proposed not only to regulate differential transport of a wide range of cargos, but also to completely prevent fouling ${ }^{19}$. Most interestingly, micropores between air sacs in the lung are filled with liquid that has been proposed to reversibly reconfigure into an open, fluid-lined pore in response to pressure gradients ${ }^{20}$.

Figure 1 contrasts the gating mechanisms in a traditional and in a liquid-filled pore. In the case of traditional nano/micropores (Fig.1a), gases will flow through passively regardless of 
pore shape and surface chemistry, while liquids will enter the pore once the applied pressure reaches a critical value dictated by the balance of surface interactions, pore geometry and surface tension. $^{21-23}$ If pores are filled with a strongly wetting liquid that completely seals the pore and forms a contiguous coating along the adjacent surface (Fig.1b), gases and liquids must deform the surface of this liquid to enter the pore and will require different pressure thresholds to do so. As long as the pore liquid's affinity for the solid is stronger than that of the transport substance, the pore liquid will part to form an open, fluid-lined pathway while remaining adherent to the pore walls and adjacent surface so that the transport substance, as long as it is immiscible, will not contact any solid surfaces. Unlike with bare pores, the transport substance will thus see only the fluid in either the open or closed states, preventing fouling both inside ${ }^{24}$ and around ${ }^{25}$ the pore. Because this dynamic gating mechanism involves structural reconfiguration rather than expulsion of the pore liquid, the pore will stay open only as long as the transport substance is flowing and will be thermodynamically primed to close as soon as the pressure drops below the threshold.

The dynamic gating mechanism depends on fluid miscibility and fluid-surface interactions rather than on specific solid or fluid properties, so it can be used to design gated transport systems using a wide variety of pore sizes, pore geometries, surface chemistries and gating liquids. We illustrate this by using membrane materials having different pore structures (Fig.2a, Fig.S1) and chemistries, ranging from hydrophobic polytetrafluoroethylene (PTFE), poly(vinylidene fluoride) (PVDF), and polypropylene (PP) (Table S1) to hydrophilic nylon. Air flows through all of these materials in their unmodified state at zero pressure, but adding a gating liquid creates a substantial critical gating pressure that has a distinct value for each membrane (Fig.2b). Since the liquid-gated pore can open and close in response to different conditions and transport substances, gas and liquid flow can be differentially controlled in a single system. For example, filling PTFE pores with a low-surface-energy liquid simultaneously generates a gating pressure for air and alters - in this case, lowers - the critical pressure for water so that both substances can be transported through the membrane in succession according to their distinct thresholds (Fig.2c, Fig.S2).

Gating for gases and liquids is expected to occur via the same capillary mechanism, where the critical pressure will be the pressure needed to deform the surface of the pore-filling liquid (Fig.2d, insets). For a gas, the pressure that must be overcome is the Laplace pressure, $4 \gamma_{l a} / d_{e}$, where $\gamma_{l a}$ is the surface tension of the pore-filling liquid and $d_{e}$ is the average effective pore size. ${ }^{26}$ These parameters are sufficient to predict the experimentally observed critical pressure for air in a $5 \mu \mathrm{m}$ porous membrane infused with a low-surface-energy liquid (Fig.2d, green). For a liquid, the gating pressure will depend on the pore size $d_{e}$ and on the liquid-liquid interfacial tension $\gamma_{l l}^{27,28}$; in fact, this relationship is used to characterize membrane porosity by observing the irreversible expulsion of the pore-filling liquid ${ }^{26,28}$. But in our case, where we aim not only for deformation of the surface of the transport liquid but for it to continuously flow through the reversibly reconfigurable fluid-lined pore, the working pressure will also depend on the flow rate $\mathrm{Q}$ and viscosity $\mu$ of the transport fluid, as $\Delta P \sim Q \mu / k^{29}$. Here $k$ is the permeability of the membrane, which is related to the pore structure and size and also depends on the

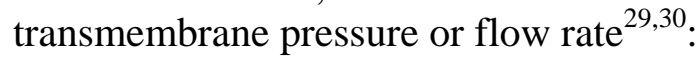

$$
k=\frac{}{32^{2}} \int_{\frac{2 H}{P}}^{\infty} \frac{X^{2}}{\sqrt{2}} e^{\frac{(X d)^{2}}{2^{2}}} d X,
$$


where $\Phi$ is the porosity, $\tau$ the tortuosity, $d$ the mean pore size, and $\sigma$ the standard deviation of a porous membrane with distributed pore sizes (see Supplementary Information for detailed discussion). This relationship accurately predicts the gating pressure for water at a series of flow rates, both with and without the pore-filling liquid (Fig.2d, blue and red), and allows us to quantitatively determine how the performance of the system depends on the pore size, geometry, and gating liquid properties (Fig.S3, Tables S2-S3).

We can use PVDF, PP and nylon membranes with their different pore sizes to tune the absolute and relative critical pressures for gases and liquids over at least two orders of magnitude, from less than $10 \mathrm{kPa}$ to more than $200 \mathrm{kPa}$ (Fig.2e, Fig.S4). For a given membrane material and pore size, the gating pressure is finely tuned by using pore-filling liquids with systematically varying surface tension (Fig.2f, Fig.S5, Table S4). We can even adjust the system so that the critical pressure for air exceeds that for the liquid ethanol (Fig.2g). In all cases, the observed gating thresholds are independent of whether gas or liquid is flowed first (Fig.S6), and are stable over time and after cyclic alteration of gas and liquid (Fig.S7).

This combination of differential tunability and reversible opening and closing enables fast and repeated control over multiphase flows in both microfluidic and macrofluidic systems by simply adjusting the system pressure. For example, we can produce distinct air/water streams by incorporating a liquid-gated porous membrane into a port in a microfluidic channel and setting the critical pressures for air and water to $2.3 \mathrm{kPa}$ and $9.8 \mathrm{kPa}$ (Fig.3a and Fig.S8-S11, Movie S1). Below $2.3 \mathrm{kPa}$, neither substance flows through the port (Fig.3a, first panel). Between the two critical pressures, only air flows through the port, and gas-free water continues past it (Fig.3a, second panel). Above $9.8 \mathrm{kPa}$, both air and water cross the port (Fig.3a, third panel), with their precise balance responding to graded pressure changes (Fig.3a, fourth panel) since pressure increases the flow rate through the port significantly for liquids but only negligibly for gases (as derived in Supporting Information and Fig.S12). The robustness of the gating behavior indicates that the pores can sustain many rapid opening/closing cycles while remaining faithful to the original gating pressures, allowing the system to be operated continuously for at least several days (Movie S1). We also explore more complex fluid handling using these principles: we use a liquid-gated membrane and set a distinct threshold for each component of a three-phase gas/liquid/liquid mixture, and then use pressure to actively adjust and control fluid flow through the membrane to collect the different phases while no component escapes (Fig.3b, Fig.S13).

The liquid-gating strategy further suppresses fouling, as illustrated by the real-time confocal images in Fig. 4a that show that a Rhodamine B dye solution leaves no trace on fluidgated membranes once the flow stops while bare pores retain substantial dye (see also Fig.S14S15). Lining membrane pores and outer surface with fluid prevents thus indeed the transport substance from contacting the solid (Fig.1), enabling the separation of suspensions containing particles larger than the pore diameter without risk of fouling. This is further illustrated in Fig 4b, where salt particles are found only on top of the closed pores following flow and are collected by a gentle surface rinse, while the bare membrane traps the particles in and around the pores and resists repeated rinsing (see also Fig.S16). The integration of antifouling behavior and pressurecontrolled flow properties in one system not only provides the flexibility to have a lower working pressure for liquid transport than with conventional membranes, but also avoids the common problem of working pressure build-up caused by pore clogging and uncontrolled fouling. Both effects can lead to significant energy savings over long-term operation (Fig.4c, see Supplementary Information for discussion). 
Fluid lining prevents fouling not only inside a nano- or micropore, but also in much longer microfluidic channels. As illustrated in Fig.4d, a microfluidic channel lined with a fluidfilled porous membrane shows no retention of Rhodamine B, fluorescent microparticles, fluorescent protein or blood, whereas state-of-the-art but conventional non-fouling microfluidic channels retain substantial residue. By using fluid lining, it should therefore be possible to design microfluidic systems that resist fouling and enable tunable gated flow of complex multiphase substances.

The dynamic reconfiguration of a liquid lining as a means to reversibly open and close fluid-lined membrane pores provides an attractive and tunable gating mechanism, which that integrates chemical and physical selectivity for gas- or liquid-phase transport substances with non-fouling behavior and energy-efficient operation. We anticipate that these capabilities, combined with the longevity of the membrane systems and adaptability for a range of materials, geometries and both macro- and microfluidic operation, will find use in many applications ranging from water treatment and biomedical fluid processing to $3 \mathrm{D}$ printing and microscale reactor operation and beyond.

\section{References}

1 Chen, P. C. \& Xu, Z. K. Mineral-Coated Polymer Membranes with Superhydrophilicity and Underwater Superoleophobicity for Effective Oil/Water Separation. Sci. Rep. 3, 2776 (2013)

2 Holt, J. K. et al. Carbon nanotube-based membranes: A platform for studying nanofluidics. 2004 4th Ieee Conference on Nanotechnology, 110-112 (2004)

3 Peng, X. S., Jin, J., Nakamura, Y., Ohno, T. \& Ichinose, I. Ultrafast permeation of water through protein-based membranes. Nat. Nanotechnol. 4, 353-357 (2009)

4 Paven, M. et al. Super liquid-repellent gas membranes for carbon dioxide capture and heartlung machines. Nat. Commun. 4, 2512 (2013)

5 Faulkner-Jones, A. et al. Development of a valve-based cell printer for the formation of human embryonic stem cell spheroid aggregates. Biofabrication 5, 015013 (2013)

6 Oh, K. W. \& Ahn, C. H. A review of microvalves. J. Micromech. Microeng. 16, R13 (2006)

7 Kargov, A. et al. Development of an anthropomorphic hand for a mobile assistive robot. 2005 Ieee 9th International Conference on Rehabilitation Robotics, 182-186 (2005)

8 Edwards, D. A. et al. Large porous particles for pulmonary drug delivery. Science 276, 1868-1871 (1997)

9 Kralj, J. G., Sahoo, H. R. \& Jensen, K. F. Integrated continuous microfluidic liquid-liquid extraction. Lab Chip 7, 256-263 (2007)

10 Karlsson, J. M. et al. Active liquid degassing in microfluidic systems. Lab Chip 13, 4366$4373(2013)$

11 Ulbricht, M. Advanced functional polymer membranes. Polymer 47, 2217-2262 (2006)

12 Lin, N. H., Kim, M. M., Lewis, G. T. \& Cohen, Y. Polymer surface nano-structuring of reverse osmosis membranes for fouling resistance and improved flux performance. J. Mater. Chem. 20, 4642-4652 (2010)

13 Powell, M. R., Cleary, L., Davenport, M., Shea, K. J. \& Siwy, Z. S. Electric-field-induced wetting and dewetting in single hydrophobic nanopores. Nat. Nanotechnol. 6, 798-802 (2011) 
14 Yameen, B. et al. Ionic transport through single solid-state nanopores controlled with thermally nanoactuated macromolecular gates. Small 5, 1287-1291 (2009)

15 Wen, Y. Q. et al. DNA-based intelligent logic controlled release systems. Chem. Commun. 48, 8410-8412 (2012)

16 Adrus, N. \& Ulbricht, M. Novel hydrogel pore-filled composite membranes with tunable and temperature-responsive size-selectivity. J. Mater. Chem. 22, 3088-3098 (2012)

17 Nair, R. R., Wu, H. A., Jayaram, P. N., Grigorieva, I. V. \& Geim, A. K. Unimpeded permeation of water through Helium-Leak-Tight graphene-based membranes. Science 335, 442-444 (2012)

18 Stroock, A. D., Pagay, V. V., Zwieniecki, M. A. \& Holbrook, N. M. The physicochemical hydrodynamics of vascular plants. Annu. Rev. Fluid. Mech. 46, 615-642 (2014)

19 Peleg, O. \& Lim, R. Y. H. Converging on the function of intrinsically disordered nucleoporins in the nuclear pore complex. Biol. Chem. 391, 719-730 (2010)

20 Namati, E., Thiesse, J., de Ryk, J. \& McLennan, G. Alveolar dynamics during respiration: are the pores of Kohn a pathway to recruitment? Am. J. Respir. Cell Mol. Biol. 38, 572-578 (2008)

21 Winther-Jensen, B., Winther-Jensen, O., Forsyth, M. \& Macfarlane, D. R. High rates of oxygen reduction over a vapor phase-polymerized PEDOT electrode. Science 321, 671-674 (2008)

22 Mohns, J. \& Kunnecke, W. Flow-Analysis with membrane separation and time-based sampling for ethanol determination in beer and wine. Anal. Chim. Acta 305, 241-247 (1995)

23 Liu, C. C., Thompson, J. A. \& Bau, H. H. A membrane-based, high-efficiency, microfluidic debubbler. Lab Chip 11, 1688-1693 (2011)

24 Yusko, E. C. et al. Controlling protein translocation through nanopores with bio-inspired fluid walls. Nat. Nanotechnol. 6, 253-260 (2011)

25 Wong, T. S. et al. Bioinspired self-repairing slippery surfaces with pressure-stable omniphobicity. Nature 477, 443-447 (2011)

26 Mietton-Peuchot, M., Condat, C. \& Courtois, T. Use of gas-liquid porometry measurements for selection of microfiltration membranes. J. Membrane Sci. 133, 73-82 (1997)

27 Zhang, C. Y., Oostrom, M., Wietsma, T. W., Grate, J. W. \& Warner, M. G. Influence of viscous and capillary forces on immiscible fluid displacement: pore-scale experimental study in a water-wet micromodel demonstrating viscous and capillary fingering. Energy Fuels 25, 3493-3505 (2011)

28 Germic, L. et al. Characterization of polyacrylonitrile ultrafiltration membranes. $J$. Membrane Sci. 132, 131-145 (1997)

29 Biot, M. A. General theory of three - dimensional consolidation. J. Appl. Phys. 12, 155-164 (1941)

30 Purcell, W. R. \& Co., S. O. Capillary pressures - their measurement using mercury and the calculation of permeability therefrom. J. Petrol. Technol. 1, 39-48 (1949)

Supplementary Information is linked to the online version of the paper at www.nature.com/nature.

\section{Acknowledgements}


This work was supported in part by the Advanced Research Projects Agency-Energy (ARPA-E), U.S. Department of Energy, under Award Number DE-AR0000326. The authors thank M. Aizenberg, R.T. Blough and X.Y. Chen for discussion; A.B. Tesler for assistance with the scanning electron microscopy; T.S. Wong, B.D. Hatton and R.A. Belisle for assistance with antifouling experiments.

\section{Author contributions}

X.H. and J.A. designed the liquid-infused porous materials and the experiments. X.H. and M.K. carried out the experiments. X.H., A.G., M.K., Y.H.H. and J.A. analyzed data. Y.H.H. built the mathematical model. All authors interpreted data and wrote the paper.

\section{Author information}

Reprints and permissions information is available at www.nature.com/reprints. The authors declare no competing financial interests. Correspondence and requests for materials should be addressed to jaiz@seas.harvard.edu.

\section{Figure legends}

Figure 1 | Hypothesis for gating a pore by liquid reconfiguration. a, For a solid nano/micropore (significantly larger than molecular scale), transport of gases (light gray) is uncontrolled and occurs even at zero pressure, while transport of liquids (red) depends on the meniscus formation defined by the interactions with the solid surface and therefore occurs at specific finite pressure. The system is prone to fouling. b. If the pore is filled with a stably held liquid (green), flow of both gases and liquids will be gated by pressure-induced deformation of the liquid interface. In the open state, the gating liquid will reversibly reconfigure to form a fluid-lined pore. Each transport substance will have a specific critical pressure based on its ability to overcome the capillary pressure at the liquid-gas or liquid-liquid interface, and the fluid-lined pore will prevent contact with the solid. When the pressure is released, a non-fouled pore returns to its original liquid-filled state. The liquid-based gating mechanism provides a unified strategy for selective, responsive, tunable, and antifouling multiphase transport.

Figure 2 | Design and rational tuning of gating systems with differentially controlled gas and liquid transport. a, Left: Scanning electron micrograph of a typical porous membrane. Scale bar is $5 \mu \mathrm{m}$. Right: Schematic of pressure measurement setup. b. Critical pressure required for gas to flow through porous materials of various chemistries and pore structures (average pore size $0.45 \mu \mathrm{m}$ ), with and without a gating liquid. For each material, $P_{\text {critical(gas }}=0$ without the gating liquid and $P_{\text {critical(gas) }}>0$ with the gating liquid. Error bars shown for each data point in b, d, e and $\mathrm{f}$ represent standard deviation. c, Liquid-based gating creates different critical pressures for gas and water within a single system. Importantly, the critical pressure for water transport in a liquid-filled membrane is reduced compared to the same dry membrane. d, Insets: Schematic model for determining critical pressure for gases and liquids. In both cases, the gating pressure is a function of the pore geometry and interfacial (gas-liquid or liquid-liquid) tension. Graph: Predictive model (lines) agrees with experimentally obtained critical pressures (circles) over a range of flow rates for air through liquid-gated pores (green), water through liquid-gated pores (blue), and water through pores without a liquid gate (red). e, Differential tuning of absolute and relative critical pressures for air and water to flow through liquid-gated pores with different pore sizes. f, Systematic tuning of the gating threshold for air transport by infusing porous nylon membranes with a series of gating liquids of different surface tensions, achieved by mixing water and ethanol in different ratios. $\mathbf{g}$, Critical pressures for air (black) and ethanol (red) through liquid-gated pores. The difference in interfacial 
tensions leads to a unique situation when the critical pressure for liquid is lower than that for gas. Inset: Without the gating liquid, the critical pressures for both air and ethanol are negligible.

Figure 3 | Sorting of multiphase mixtures by liquid-gated pores. a, Rapid pressure-tunable sorting of multiphase flows in a microfluidic system. Top: schematics; Bottom: snapshots of experimental data. A liquid-infused porous material incorporated into a port along a microfluidic channel enables a series of distinct pressure-dependent scenarios for a mixed gas and liquid flow. In schematics, the membrane is shown in green, and the transported liquid in blue; the blue arrows depict the transport of liquid, dashed black and blue arrows correspond to the transport of a mixed gas/liquid phase, and dashed black and white arrows depict the transport of gas. First panel: At pressures below both $P_{\text {critical(gas) }}$ and $P_{\text {critical(liquid), }}$ nothing flows through the port. Second panel: Above $P_{\text {critical(gas) }}$ and below $P_{\text {critical(liquid), }}$ only the gas flows through the port and degassed liquid continues through the channel beyond the port. Third and fourth panels: At pressures above both $P_{\text {critical(gas) }}$ and $P_{\text {critical(liquid), both phases cross the port and only liquid }}$ continues through the channel beyond the port. Note that above both critical pressures, the liquid:gas ratio that crosses the port increases with increasing pressure. Data shown are for an alternating air-liquid flow, with $P_{\text {critical(gas) }}=2.3 \mathrm{kPa}$ and $P_{\text {critical(liquid) }}=9.8 \mathrm{kPa}$. $\mathbf{b}$, A three-phase mixture of air, water and crude oil is progressively separated by the liquid-infused porous membrane.

Figure 4 | Antifouling transport and separation of complex substances. a, Real-time confocal images of a liquid-infused porous material (top) or of the porous material without the gating liquid (bottom) before, during, and after flowing an aqueous solution of Rhodamine B dye (RB). RB retention after flow is observable only in the PTFE membrane without the gating liquid. b, Flowing a suspension of 4Benzoylamino-2,5-diethoxybenzenediazonium particles $(1000 \mu \mathrm{L} / \mathrm{min})$ through a liquid-infused porous material (top) leaves salt particles suspended on the liquid surface after the pore closes (top, center), and the particles are easily collected by a gentle surface rinse (top, right). The bare membrane (bottom) traps the salts both in and around the pores (bottom, center) and resists repeated rinsing (bottom, right). c, Pressure for transporting a suspension of 4-Benzoylamino-2,5-diethoxybenzenediazonium particles in water through a liquid-infused PTFE membrane is initially $38.7 \%$ lower than through the dry membrane. This value increases to about $56.5 \%$ after $4.5 \mathrm{~h}$ operation at a flow rate of $50 \mu \mathrm{L} / \mathrm{min}$ due to the fouling of the dry membrane. The energy saving rate $=\left(P_{\mathrm{PTFE}}-P_{\mathrm{PTFE}}\right.$ with Krytox®103 $) / P_{\mathrm{PTFE}} * 100 \%$. d, Liquid-lined PTFE microchannel resists fouling (top) by RB solution, suspended microparticles, protein solution, (fluorescent images) and blood (optical image), while fouling residue is observed inside the bare PTFE channel for each substance (bottom). 
a

\section{Pore}

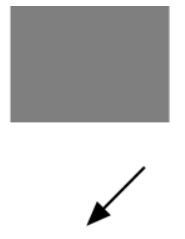

Gas

ป

$P_{\text {critical (gas) }}=0$ b

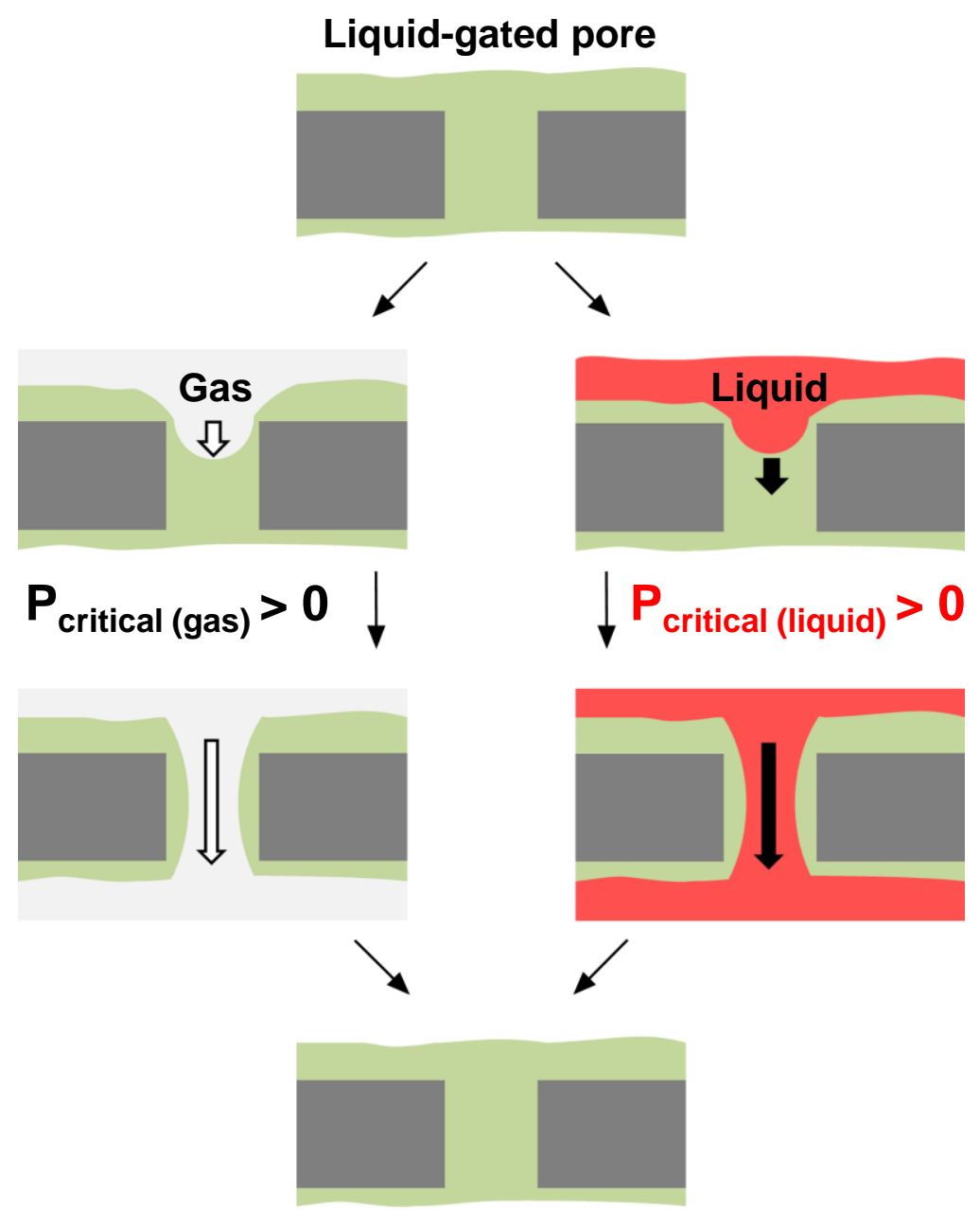


a

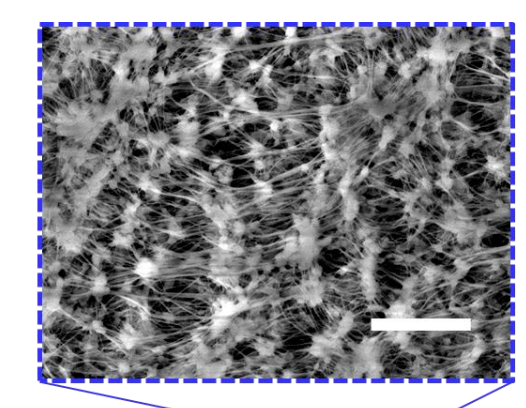

Porous membrane Porous membrane
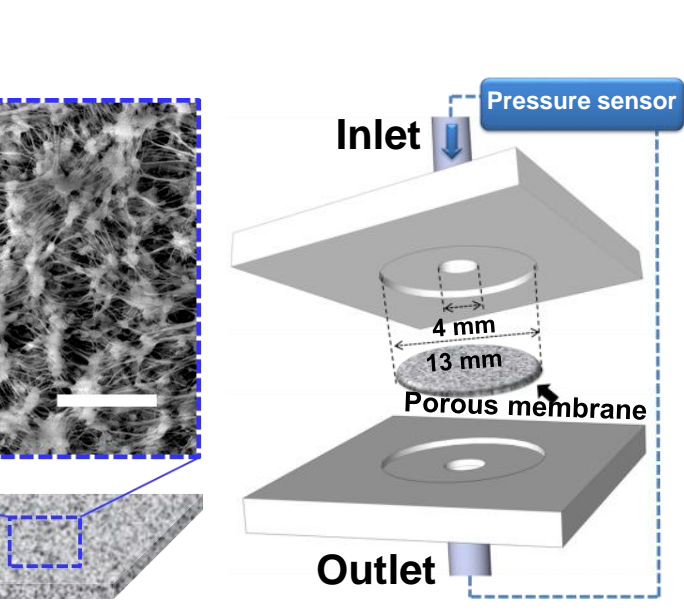

b
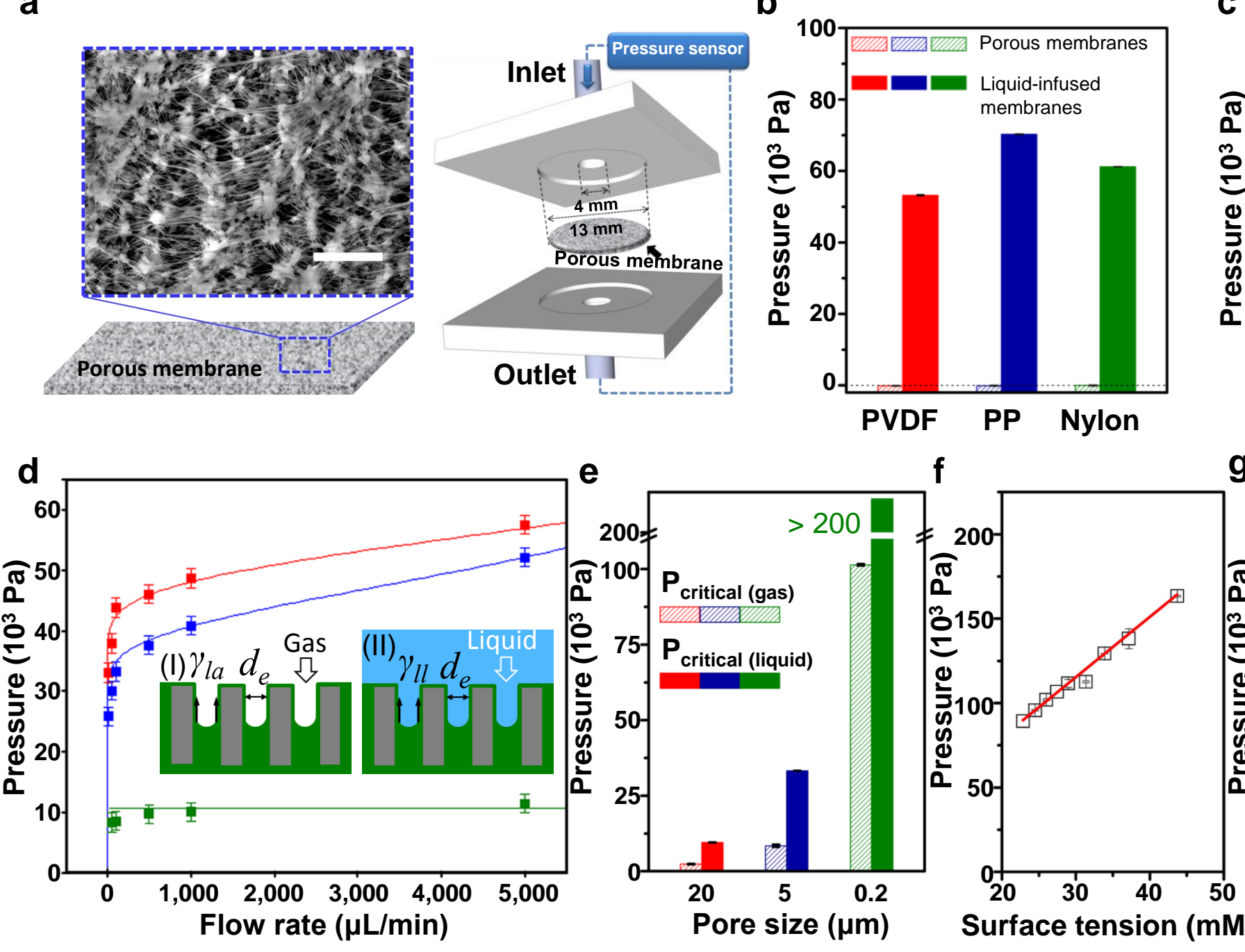

Surface tension $(\mathrm{mM} / \mathrm{m})$

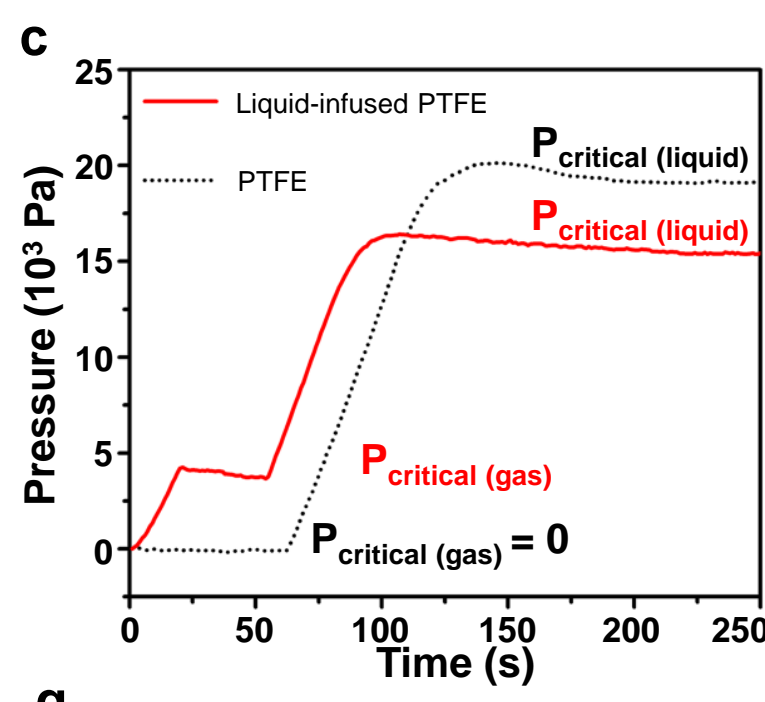

g

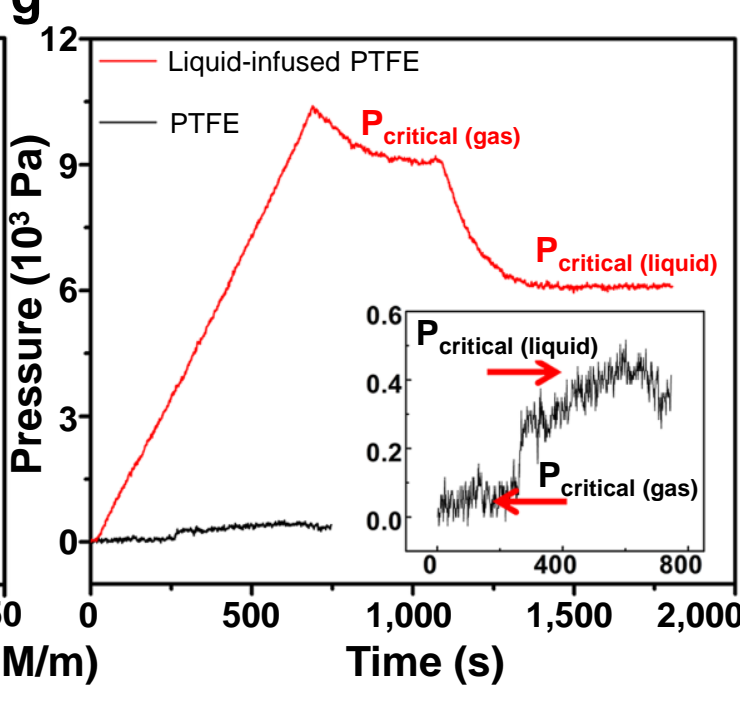


a $\quad \Delta \mathbf{P}<\mathbf{P}_{\text {critical(gas) }}<\mathbf{P}_{\text {critical(liquid) }}$

$\mathbf{P}_{\text {critical(gas) }}<\Delta \mathbf{P}<\mathbf{P}_{\text {critical(liquid) }}$

$\mathbf{P}_{\text {critical(gas) }}<\mathbf{P}_{\text {critical(liquid) }}<\Delta \mathbf{P} \quad \mathbf{P}_{\text {critical(gas) }}<\mathbf{P}_{\text {critical(liquid) }} \ll \Delta \mathbf{P}$
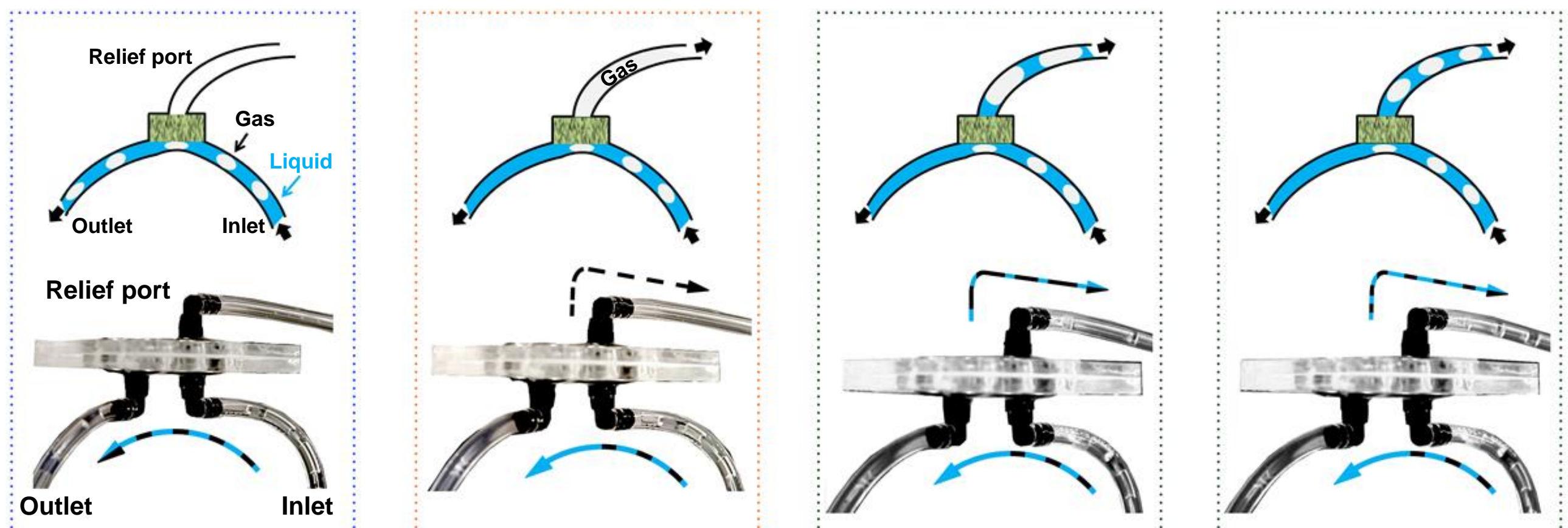

b

Liquid-infused PTFE

Air/water/oil mixture:

Air bubble

Water Oil $\mathbf{P}<\mathbf{P}_{\text {(gas) }}<\mathbf{P}_{\text {(water) }}<\mathbf{P}_{(\text {oil) }}$ $\mathbf{P}_{\text {(gas) }}<\mathbf{P}<\mathrm{P}_{\text {(water) }}<\mathrm{P}_{\text {(oil) }}$ $\mathbf{P}_{\text {(gas) }}<\mathbf{P}_{\text {(water) }}<\mathbf{P}<\mathrm{P}_{\text {(oil) }}$

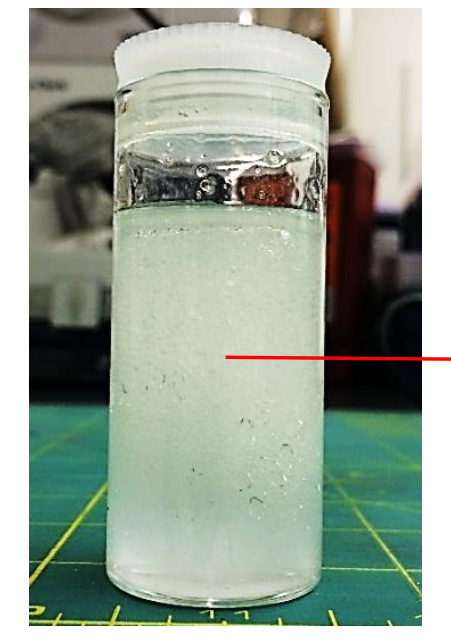

Air/water/oil mixture

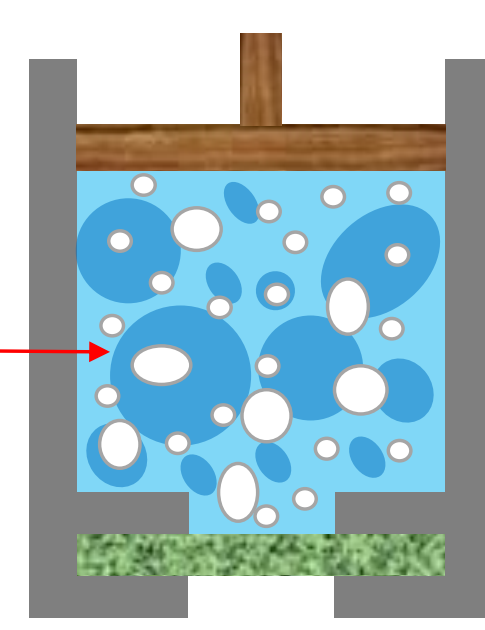

Outlet

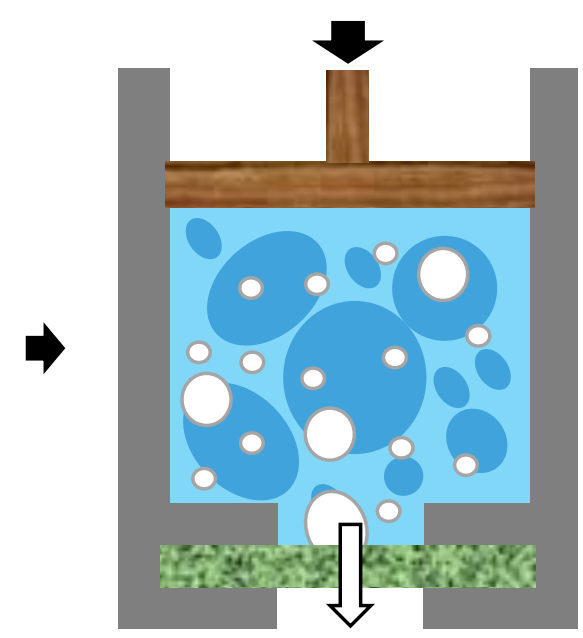

Air

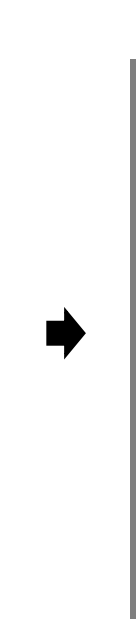

$c$

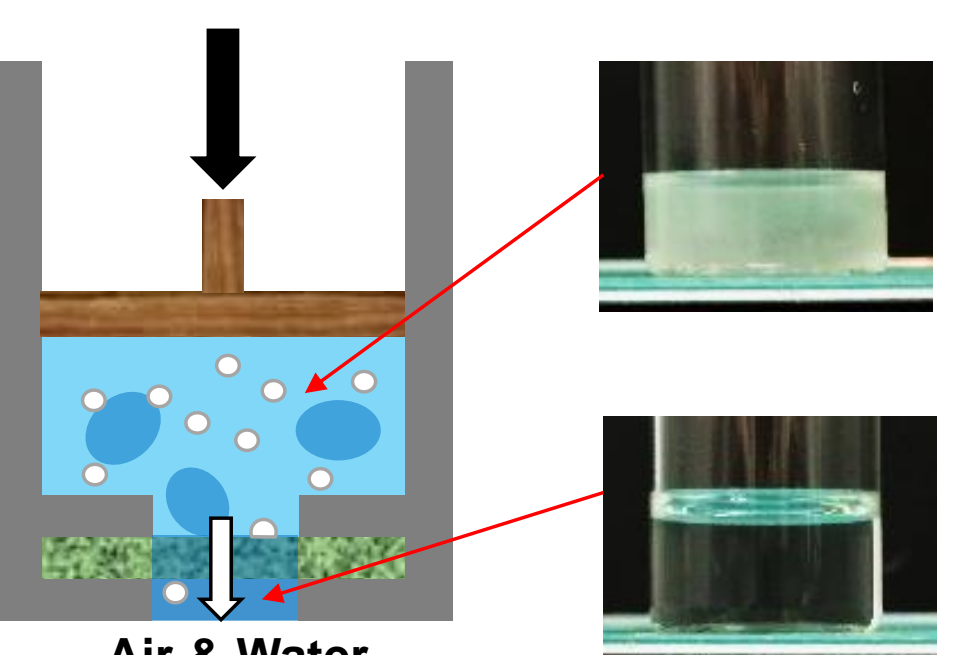


Liquid-infused membrane $\downarrow$ RB

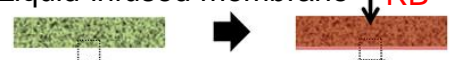

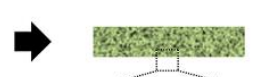

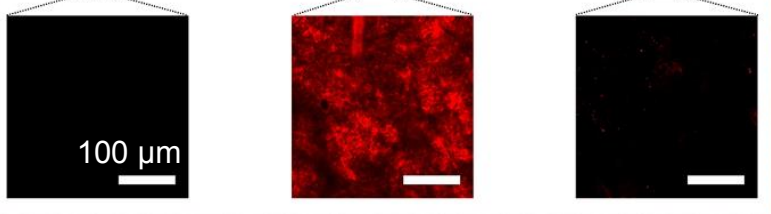

$\Rightarrow \quad \downarrow$

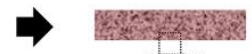

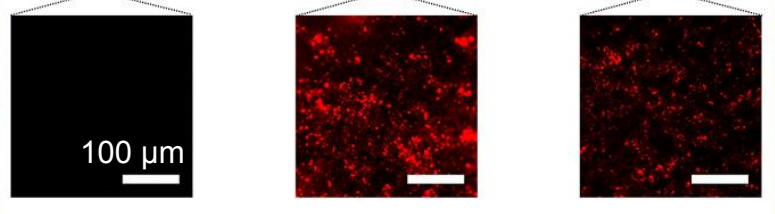

b

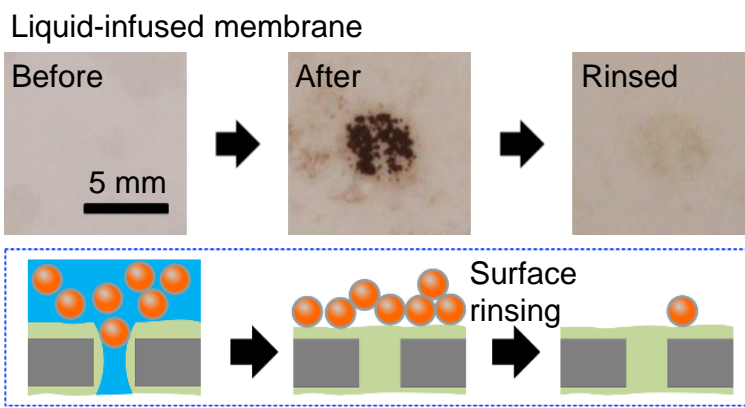

Membrane

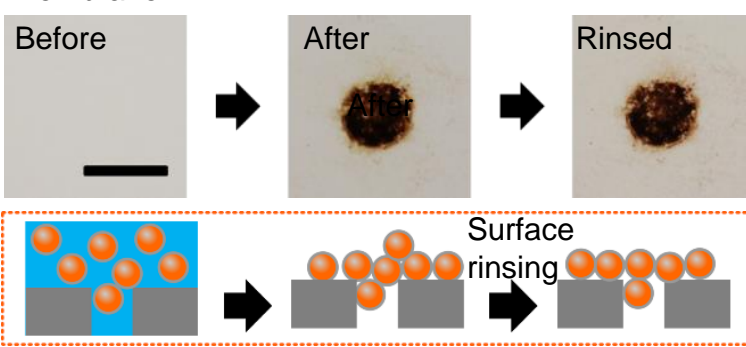

d

Microchannels

$$
\text { C }
$$
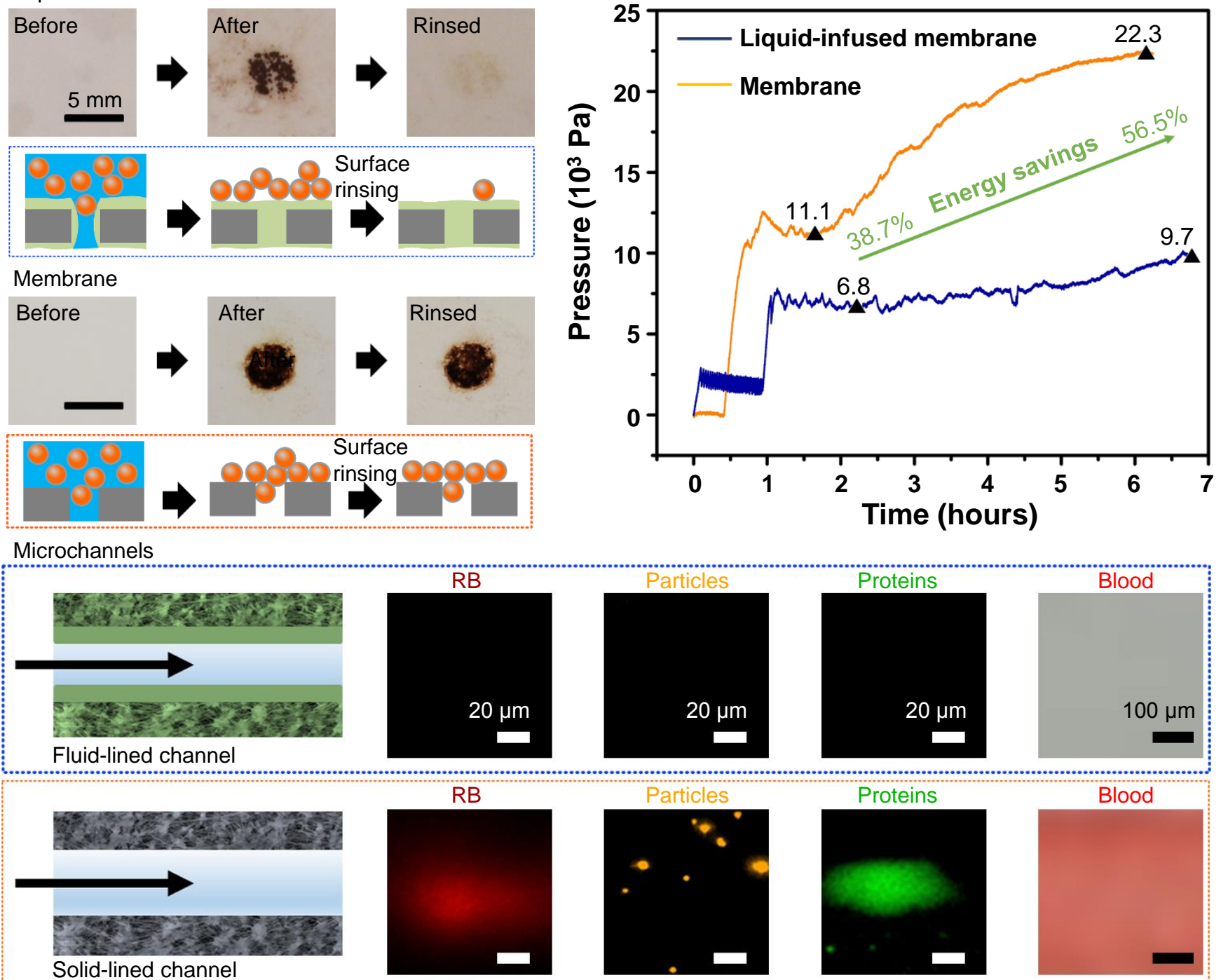